\title{
Reducing disorder-induced losses for slow light photonic crystal waveguides through Bloch mode engineering
}

Mann, Nishan; Combrie, Sylvian; Colman, Pierre; Patterson, Mark; De Rossi, Alfredo; Hughes, Stephen

Published in:

Optics Letters

Link to article, DOI:

10.1364/OL.38.004244

Publication date:

2013

Document Version

Publisher's PDF, also known as Version of record

Link back to DTU Orbit

Citation (APA):

Mann, N., Combrie, S., Colman, P., Patterson, M., De Rossi, A., \& Hughes, S. (2013). Reducing disorderinduced losses for slow light photonic crystal waveguides through Bloch mode engineering. Optics Letters, 38(20), 4244-4247. https://doi.org/10.1364/OL.38.004244

\section{General rights}

Copyright and moral rights for the publications made accessible in the public portal are retained by the authors and/or other copyright owners and it is a condition of accessing publications that users recognise and abide by the legal requirements associated with these rights.

- Users may download and print one copy of any publication from the public portal for the purpose of private study or research.

- You may not further distribute the material or use it for any profit-making activity or commercial gain

- You may freely distribute the URL identifying the publication in the public portal 


\title{
Reducing disorder-induced losses for slow light photonic crystal waveguides through Bloch mode engineering
}

\author{
Nishan Mann, ${ }^{1, *}$ Sylvian Combrié, ${ }^{2}$ Pierre Colman, ${ }^{3}$ Mark Patterson, ${ }^{1}$ Alfredo De Rossi, ${ }^{2}$ and Stephen Hughes ${ }^{1}$ \\ ${ }^{1}$ Department of Physics, Queen's University, Kingston, Ontario K7L 3N6, Canada \\ ${ }^{2}$ Thales Research and Technology, Route Departementale 128, 91767 Palaiseau Cedex, France \\ ${ }^{3}$ DTU Fotonik, Orsted Plads, Kongens, Lyngby, Denmark \\ *Corresponding author: nmann@physics.queensu.ca
}

Received July 1, 2013; revised August 27, 2013; accepted September 4, 2013; posted September 6, 2013 (Doc. ID 193109); published October 14, 2013

\begin{abstract}
We present theory and measurements of disorder-induced losses for low loss $1.5 \mathrm{~mm}$ long slow light photonic crystal waveguides. A recent class of dispersion engineered waveguides increases the bandwidth of slow light and shows lower propagation losses for the same group index. Our theory and experiments explain how Bloch mode engineering can substantially reduce scattering losses for the same slow light group velocity regime. @ 2013 Optical Society of America

OCIS codes: (290.4210) Multiple scattering; (130.5296) Photonic crystal waveguides.

http://dx.doi.org/10.1364/OL.38.004244
\end{abstract}

Photonic crystal waveguides (PCWs), created by introducing a line defect in a high-index contrast photonic crystal (PC) slab, permit strongly confined light to be guided on subwavelength scales. In particular, PCWs exhibit a "slow light" region in their band structure. Slow light behavior enhances light-matter interactions that have various applications such as optical data processing [1] and creating on-chip single photons [2]. Recently, there has been much interest in exploiting slow light propagation with PCWs for enhancing nonlinear optical processes, including second and third harmonic generation [3,4], four wave mixing $[5,6]$ and three photon absorption [7].

One of the most serious limitations of using slow light PCWs is extrinsic scattering losses caused by manufacturing imperfections in the underlying dielectric structure of the PC $[8,9]$. Fabrication imperfections are characterized by small fluctuations $(2-4 \mathrm{~nm})$ of the radius of the holes that act as extrinsic polarization scattering sites. In the slow light regime, the Bloch modes feel these scatterers much more due to their increased optical path length and an increase in the local density of states. Thus, the slow light regime exhibits large scattering losses that have been observed experimentally $[\underline{9}, \underline{10}]$ and explained theoretically [8,11-14]. For a fixed group velocity, the underlying Bloch mode affects the amount of scattering loss [8]. To better exploit slow light, one can tailor the dispersion via geometrical modifications of the underlying periodical dielectric structure, a process sometimes referred to as "dispersion engineering." Two common goals of dispersion engineering (DE) are (i) to minimize group velocity dispersion (GVD) over a finite range of frequencies [15], giving one a better delay bandwidth product; and (ii) to design slow light regions away from the mode edge. Recently, Sancho et al. [16] have designed a PCW to minimize GVD that has also been observed to yield lower disorder-induced losses relative to the standard W1 waveguide (i.e., a simple line defect created by removing one row of holes in a triangular lattice). In addition, the loss characteristics of this dispersion engineered waveguide deviate strongly from the expected $1 / v_{g}^{2}$ or $1 / v_{g}$ group velocity scaling, which can be caused by a reshaping of the Bloch mode and (or) a failure of the Beer-Lambert law due to multiple scattering [17]. O'Faolain et al. [18] have also confirmed, theoretically, that certain $\mathrm{PCW}$ s can have different Bloch modes for the same group velocity.

In this work, we exploit and extend a rigorous disorder model based on the work of Hughes et al. [8] to obtain good qualitative agreement with experiments performed on PCWs with various dispersion-engineered designs. We also explain the recent experimental data of Sancho et al. [16]. Without doing any optimization, we demonstrate that our dispersion-engineered waveguides already reduce transmission losses by a factor of 2 compared to the standard W1 for a slow down factor (group index) of $n_{g}=20$. We present a good fit to the data over a wide spectral range with no fitting parameters and show that it is mainly the field strength of the Bloch mode around the holes that is responsible for the lower losses. Moreover, our general methodology offers a way to design lower loss waveguides operating in the slow light regime.

Disorder-induced losses in PCWs can be divided into backscattering and radiation losses, with backscattering being the dominant loss mechanism [8]. The ensemble averaged backscattering loss, per unit cell, is [8]

$$
\left\langle\alpha_{\text {back }}\right\rangle=\left(\frac{a \omega}{2 v_{g}}\right)^{2} \iint_{\text {cell }} \operatorname{drd} \mathbf{r}^{\prime}\left\langle[\mathbf{E}(\mathbf{r}) \cdot \mathbf{P}(\mathbf{r})]\left[\mathbf{E}^{*}\left(\mathbf{r}^{\prime}\right) \cdot \mathbf{P}^{*}\left(\mathbf{r}^{\prime}\right)\right]\right\rangle,
$$

where $\mathbf{E}(\mathbf{r})=\mathbf{e}_{k}(\mathbf{r}) e^{i k x}, \mathbf{e}_{k}(\mathbf{r})$ is the Bloch mode, $a$ is the pitch, $v_{g}$ is the group velocity, and $\mathbf{P}(\mathbf{r})$ represents the structural disorder. The Bloch modes are normalized through $\int_{\text {cell }} \operatorname{dr} \varepsilon(\mathbf{r})\left|\mathbf{e}_{k}(\mathbf{r})\right|^{2}=1$, where $\varepsilon(\mathbf{r})$ is the ideal dielectric constant of the PCW. The bracket term $\langle\cdots\rangle$ refers to an ensemble average over nominally identical waveguides. The polarization term describing disorder $[19,20]$ accounts for a quickly varying dielectric perturbation around the hole surfaces. The ensemble averaged radiation loss per unit cell, $\left\langle\alpha_{\mathrm{rad}}(\omega)\right\rangle$, is derived similarly [17]. For our calculations, the band structure and eigenmodes of Maxwell's equations for the fundamental unit cell 
were computed by MPB [21]. The integration is carried out using a double Riemann sum with the integrand interpolated over a finer spatial grid for good numerical accuracy.

The disorder polarization term $\mathbf{P}(\mathbf{r})$, due to a disordered element at $\mathbf{r}^{\prime}$, is given by $\mathbf{P}(\mathbf{r})=\left[\left(\varepsilon_{1}+\right.\right.$ $\left.\left.\varepsilon_{2} / 2\right) \alpha_{\|} \mathbf{E}_{\|}(\mathbf{r})+\varepsilon(\mathbf{r}) \gamma_{\perp} \mathbf{D}_{\perp}(\mathbf{r})\right] \Delta V \delta\left(\mathbf{r}-\mathbf{r}^{\prime}\right)$, where $\varepsilon_{1,2}$ are the dielectric constants for the air and the slab, respectively, $\alpha_{\|}, \gamma_{\perp}$ are the numerically computed polarizabilities representing the disordered hole, $\mathbf{E}_{\|} / \mathbf{D}_{\perp}$ are the parallel and perpendicular components of the electric/ displacement fields, respectively, and $\Delta V$ is the volume of the disorder element. The rapid intrahole radial fluctuations, $\Delta r(\theta)$, representing disorder are contained in $\Delta V[19,20]$. It can be shown that Eq. (1) is reduced to a line integral around the hole circumferences. The random radial fluctuations, $\Delta r(\theta)$, vary as a function of angle $\theta$ and are modeled as a Gaussian stochastic process that is determined by its first (mean) and second (variance $\sigma^{2}$ ) order moments. Consistent with experimental data, intrahole fluctuations are assumed to be correlated so we introduce the correlation length, $l_{c}$ [22]. The mean is assumed to be zero and the variance and correlation length are estimated from scanning electron microscope (SEM) images.

For sufficiently long waveguide lengths, multiple scattering events become significant and weak localization occurs [17]. For such cases, the Beer-Lambert loss model breaks down and overestimates the losses [23]. To account for multiple scattering when computing the transmission, we utilize incoherent coupled-mode theory [23]. Denoting $\Psi_{f}(x), \Psi_{b}(x)$ as the forward and backward Bloch mode intensities, respectively, where $x \in[0, L]$ represents the position in the PCW, the coupled mode equations are ( $\omega$ is implicit)

$$
\begin{gathered}
\frac{d \Psi_{f}}{d x}=-\left(\left\langle\alpha_{\mathrm{back}}\right\rangle+\left\langle\alpha_{\mathrm{rad}}\right\rangle\right) \Psi_{f}+\left\langle\alpha_{\mathrm{back}}\right\rangle \Psi_{b}, \\
\frac{d \Psi_{b}}{d x}=\left(\left\langle\alpha_{\mathrm{back}}\right\rangle+\left\langle\alpha_{\mathrm{rad}}\right\rangle\right) \Psi_{b}-\left\langle\alpha_{\mathrm{back}}\right\rangle \Psi_{f}
\end{gathered}
$$

With the boundary conditions, $\Psi_{f}(0)=1, \Psi_{b}(L)=0$, Eqs. (2) and (3) are solved analytically using $\left\langle\alpha_{\text {back }}\right\rangle$ and $\left\langle\alpha_{\mathrm{rad}}\right\rangle$ as input. The reflectance and transmission are given by $\operatorname{Ref}=\Psi_{b}(0)$ and Tran $=\Psi_{f}(L)$, respectively. The power loss per waveguide length $(1.5 \mathrm{~mm})$ is $P=10 \log _{10}$ (Tran) in units of $\mathrm{dB}$. The key advantages of our approach are that (a) it is semi-analytic, (b) it accounts for the local field effects from realistic quickly varying surfaces, (c) it includes multiple scattering for finite length samples, and (d) the numerical implementation is both stable and numerically efficient.
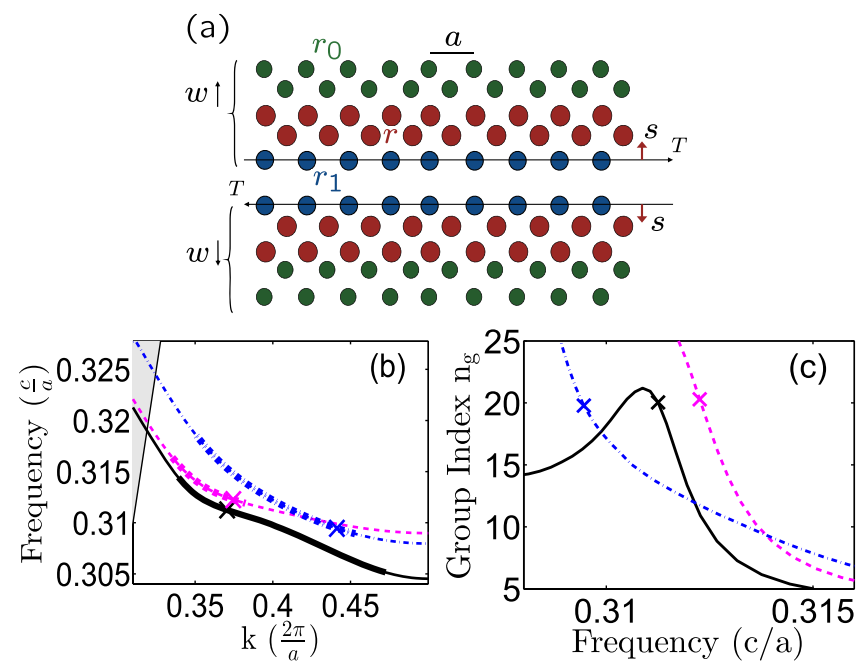

Fig. 1. (a) Schematic of the PCW designs, with the parameters given in Table 1 . The highlighted regions of the band structure in (b) are also plotted in (c) versus group index. The markers in (b) and (c) correspond to group index $n_{g}=20$, and their underlying Bloch mode intensities are plotted in Fig. $\underline{3}$.

A schematic of the template PCW design used in our experiments is shown in Fig. 1(a), where the rows of holes can be shifted and the hole radii can also change. Specific parameters are given in Table 1 . For our samples, we use GaInP dielectric membranes of length $1.5 \mathrm{~mm}$. An efficient coupler is attached to the ends of the waveguide [24]. The delay-line design was created using group theory arguments [25]. This approach exploits an antisymmetric shift ( $T$ ) of the first row of holes to couple the even and odd modes of a standard W1 waveguide. Symmetry breaking skews the Bloch modes, as shown in Fig. 3 .

Three waveguide designs are studied in this work, which we refer to as delay line, $n_{g}$-improved, and the standard W1 (see Fig. 1(a) and Table 1). In Figs. 1(b) and $1(\mathrm{c})$ we show the band structure and dispersion for the fundamental TE-like (z-even) waveguide mode of each design. The delay line was found to offer a bandwidth of $2 \mathrm{THz}$ with minimal group index $\left(n_{g}=c / v_{g}\right)$ variation compared to a $\mathrm{W} 1$ waveguide.

The loss spectrum for the waveguides is plotted in Fig. 2. For the disorder parameters, we estimate and use $\bar{\sigma} \approx 3 \mathrm{~nm}$ and $l_{c} \approx 40 \mathrm{~nm}$ to characterize the disordered holes. This figure clearly shows that the BeerLambert model breaks down and overestimates the losses in the slow light regime as expected for long (1.5 mm) PC waveguides [23]. For a given "target" group index of $n_{g}=20$, the losses are $(-9.90 \mathrm{~dB},-7.65 \mathrm{~dB}$, $-4.39 \mathrm{~dB}$ ) for the $\mathrm{W} 1$, delay line, and $n_{g}$-improved design, respectively. These results imply that the delay line and $n_{g}$-improved designs have power transmission

Table 1. Parameters for Waveguides Shown in Fig. 1(a)

\begin{tabular}{lccccccc}
\hline & $r_{0}(\mathrm{a})$ & $r_{1}(\mathrm{a})$ & $r(\mathrm{a})$ & $\mathrm{w}((\sqrt{3} / 2) a)$ & $\mathrm{s}((\sqrt{3} / 2) a)$ & $\mathrm{T}(\mathrm{a})$ & $\mathrm{h}(\mathrm{a})$ \\
\hline W1 & 0.2 & 0.2 & 0.2 & 0.0 & 0.0 & 0.0 & 0.333 \\
Delay line & 0.23 & 0.25 & 0.26 & -0.1 & 0.16 & 0.15 & 0.367 \\
$n_{g}$-improved & 0.23 & 0.25 & 0.26 & -0.1 & 0.18 & 0.0 & 0.367 \\
\hline
\end{tabular}




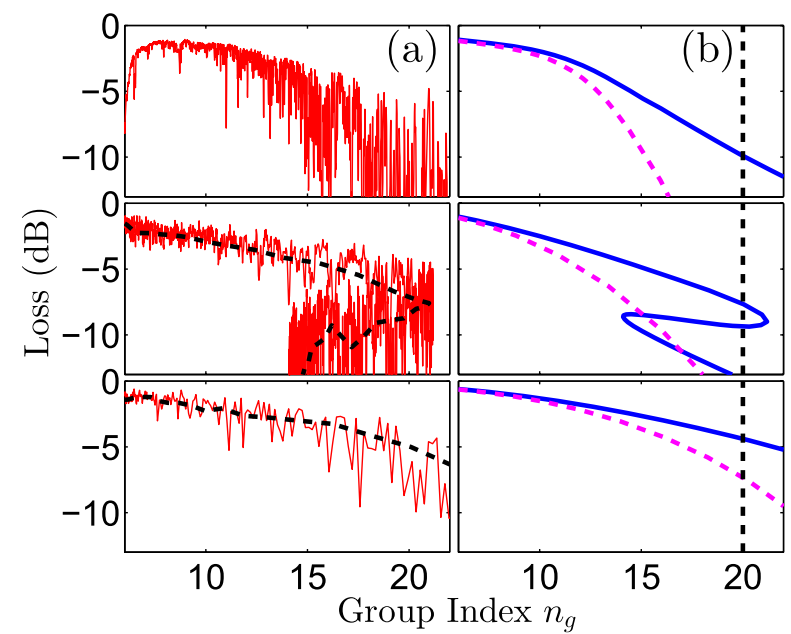

Fig. 2. Top, W1; middle, delay line; bottom, $n_{g}$-improved. The computed incoherent loss spectrum (b) with multiple scattering (blue solid) and single scattering (magenta dashed) extrapolated using the Beer-Lambert model is presented alongside experimental data (a). The experimental data includes raw data (red solid) and its statistical average (black dashed). The $n_{g}=20$ line is shown in black (vertical dashed) in (b).

approximately two times better than the $\mathrm{W} 1$ with the $n_{g^{-}}$ improved design having the lowest loss. In addition, the delay line experiences different losses for the same group index, with the losses increasing as one approaches the mode edge in agreement with the findings of O'Faolain et al. [18]. Hence, it is evident that, by comparing experiments to a self-consistent theory, waveguide design cannot only alter the dispersion characteristics but can also substantially reduce disorder-induced losses in the slow light regime. As shown in Fig. 2, the theoretical loss spectrum agrees well with the experimental loss spectrum.

Recently, it has been suggested in [18] that much longer correlation lengths on the order of the circumference of the hole should be used to obtain a better fit to certain loss spectra. This has not been the case for our calculations. The effect of correlation length on losses is nontrivial and dependent on the waveguide design. We will report on the subtle role of correlation length and related modeling details in a future publication.

To help explain the cause of three different losses for the same group index for the delay line (see Fig. 2), we point out that it is the integral term in Eq. (1) that is responsible for the different losses. The integral term involves the disorder model and the Bloch modes, which are dependent on the dispersion. A change in the localization of Bloch modes around hole boundaries has a direct effect on losses. For the W1, the Bloch mode spreads away from the line defect into the slab as one approaches the mode edge (increasing $k$ ) thereby increasing mode localization near hole boundaries, leading to enhanced losses in the slow light regime. The same pattern is present in the delay line even if the group index remains the same for three different (increasing) $(k, \omega)$ points.

To gain better insight into the amount of field localization around hole boundaries, we assume the Bloch modes do not vary significantly as a function of height of the PC slab (though these are fully accounted for in our detailed calculations). Taking a horizontal slice in the middle of slab, we introduce a simple measure for the localization of a Bloch mode around hole interfaces in the slice as $I_{f}=\sum_{i} r_{i} \int_{0}^{2 \pi}\left|\mathbf{e}_{k}\right|^{2} \mathrm{~d} \theta$, where $r_{i}$ is the radius of the $i$ th hole, $\left|\mathbf{e}_{k}\right|^{2}(\theta)$ is the field intensity on the hole boundary, and $I_{f}$ denotes the total field strength around holes in the slice and has units of length ${ }^{-2}$. The Bloch mode intensities of the W1, delay line, and $n_{g}$-improved are plotted in Fig. 3 along with $\left|\mathbf{e}_{k}^{2}\right|(\theta)$ plotted over the nearest and next-nearest hole relative to the line defect, and the total field strength is shown in the caption.

For the delay line, the skewed Bloch modes for $n_{g}=20$ result in a significant reduction of field strength along the hole boundaries compared to the $\mathrm{W} 1$ resulting in much lower loss. Over the region of the dispersion curve that has minimal group index variation the integral term dominates, resulting in a strong deviation from the expected $1 / v_{g}^{2}$ scaling, but as one approaches the mode edge the prefactor in Eq. (1) dominates and the loss spectrum has the usual $1 / v_{g}^{2}$ scaling. Recently, the delay line has been utilized to implement an integrable microwave

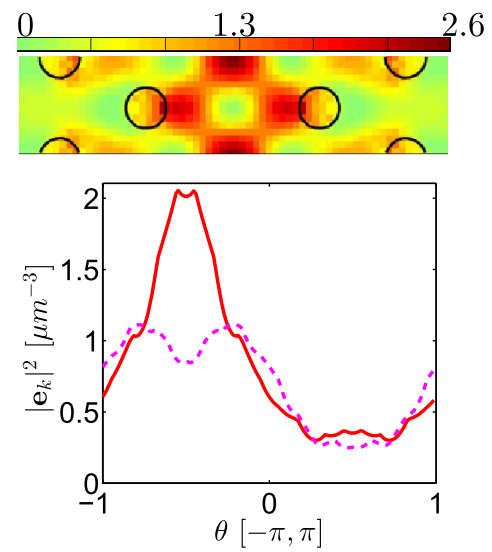

(a) W1
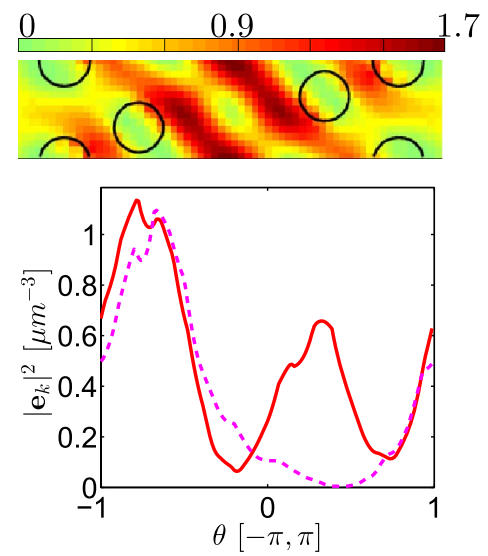

(b) Delay Line
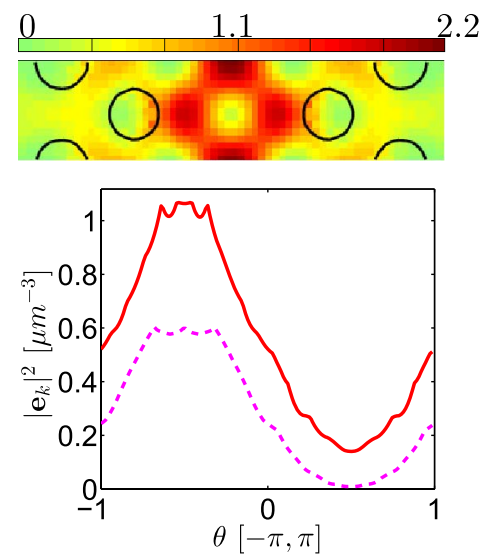

(c) $n_{g}$-improved

Fig. 3. Bloch mode intensities $\left|\mathbf{e}_{k}\right|^{2}$ at markers in Figs. 1(b) and $\underline{1(c)}$ for (a) W1, (b) delay line, and (c) $n_{g}$-improved for $n_{g}=20$ in a horizontal slice in the middle of the PC slab (top). The color scale is in units of $\mu \mathrm{m}^{-3}$. Below the field intensities in the slice at the nearest (red solid) and next nearest (magenta dashed) holes relative to the defect are shown. The total integrated field strength $I_{f}$ (units of length ${ }^{-2}$ ) around these holes is $0.9 \mu \mathrm{m}^{-2}$ (W1), $0.6 \mu \mathrm{m}^{-2}$ (delay line), and $0.6 \mu \mathrm{m}^{-2}$ ( $n_{g}$-improved). 
filter [16]. The $n_{g}$-improved design has similar field strength around hole boundaries as the delay line for $n_{g}=20$ but it has a slightly lower loss than the delay line.

Some optimization techniques have already been used to improve certain waveguide parameters such as bend loss, GVD, and the photonic band gap [26,27]. The link between field strength around hole boundaries and backscattering losses has been pointed out in simple 2D theoretical studies [28]. In addition, based on a phenomenological model for losses, Wang et al. [29] have recently obtained optimal loss engineered $2 \mathrm{D}$ PCWs. As justification for their model, they have also shown that the lowest loss implies the lowest field concentration around hole boundaries. We hypothesize that a better low loss design in $3 \mathrm{D}$ is possible by the formulation of an objective function that depends directly on the field strength around hole boundaries. Future work will introduce designs that create lower loss PCWs in the slow light regime.

In summary, using an incoherent disorder model we demonstrated good agreement with experimental data for state of the art dispersion engineered (DE) waveguides exhibiting lower losses (two times better transmission) and strong deviations from the expected group velocity scaling. Our backscatter loss expression and results explicitly show the link between minimizing field strength around holes and minimizing losses. We also show that the field strength around holes is a qualitatively good measure for predicting how losses scale for different PCW designs. Therefore, Bloch mode engineering combined with dispersion engineering is an excellent choice for minimizing losses in slow light PCWs with currently available fabrication processes.

This work was supported by the National Sciences and Engineering Research Council of Canada, the French national funding agency through the "Symphonie" grant, and Villum Fonden through the Nanophotonics for Terabit Communications (NATECA) project.

\section{References}

1. T. Krauss, Nat. Photonics 2, 448 (2008).

2. V. S. C. Manga Rao and S. Hughes, Phys. Rev. B 75, 205437 (2007).

3. K. Lenglé, L. Bramerie, M. Gay, J.-C. Simon, S. Combrié, G. Lehoucq, and A. De Rossi, Appl. Phys. Lett. 102, 151114 (2013).

4. C. Monat, M. Spurny, C. Grillet, L. O'Faolain, T. F. Krauss, B. J. Eggleton, D. Bulla, S. Madden, and B. Luther-Davies, Opt. Lett. 36, 2818 (2011).

5. J. Li, L. O'Faolain, and T. F. Krauss, Opt. Express 20, 17474 (2012).
6. C. Xiong, C. Monat, A. S. Clark, C. Grillet, G. D. Marshall, M. J. Steel, J. Li, L. O'Faolain, T. F. Krauss, J. G. Rarity, and B. J. Eggleton, Opt. Lett. 36, 3413 (2011).

7. C. Husko, S. Combrié, Q. V. Tran, F. Raineri, C. W. Wong, and A. De Rossi, Opt. Express 17, 22442 (2009).

8. S. Hughes, L. Ramunno, J. F. Young, and J. E. Sipe, Phys. Rev. Lett. 94, 033903 (2005).

9. E. Kuramochi, M. Notomi, S. Hughes, A. Shinya, T. Watanabe, and L. Ramunno, Phys. Rev. B 72, 161318 (2005).

10. L. O'Faolain, T. P. White, D. O'Brien, X. Yuan, M. D. Settle, and T. F. Krauss, Opt. Express 15, 13129 (2007).

11. D. Gerace and L. C. Andreani, Photon. Nanostr. Fundam. Appl. 3, 120 (2005).

12. B. Wang, S. Mazoyer, J. Hugonin, and P. Lalanne, Phys. Rev. B 78, 18 (2008).

13. A. Parini, P. Hamel, A. De Rossi, S. Combrié, N.-V.-Q. Tran, Y. Gottesman, R. Gabet, A. Talneau, Y. Jaouön, and G. Vadalà, J. Lightwave Technol. 26, 3794 (2008).

14. W. Song, R. A. Integlia, and W. Jiang, Phys. Rev. B 82, 235306 (2010).

15. J. Li, T. P. White, L. O'Faolain, A. Gomez-Iglesias, and T. F. Krauss, Opt. Express 16, 6227 (2008).

16. J. Sancho, J. Bourderionnet, J. Lloret, S. Combrié, I. Gasulla, S. Xavier, S. Sales, P. Colman, G. Lehoucq, D. Dolfi, J. Capmany, and A. De Rossi, Nat. Commun. 3, 1075 (2012).

17. M. Patterson, S. Hughes, S. Combrié, N.-V.-Q. Tran, A. Rossi, R. De Gabet, and Y. Jaouën, Phys. Rev. Lett. 102, 253903 (2009).

18. L. O'Faolain, S. A. Schulz, D. M. Beggs, T. P. White, M. Spasenović, L. Kuipers, F. Morichetti, A. Melloni, S. Mazoyer, J. P. Hugonin, P. Lalanne, and T. F. Krauss, Opt. Express 18, 2762738 (2010).

19. S. G. Johnson, M. L. Povinelli, M. Soljačisć, A. Karalis, S. Jacobs, and J. D. Joannopoulos, Appl. Phys. B 81, 283 (2005).

20. M. Patterson and S. Hughes, Phys. Rev. B 81, 15 (2010).

21. S. Johnson and J. Joannopoulos, Opt. Express 8, 173 (2001).

22. M. Skorobogatiy, G. Bégin, and A. Talneau, Opt. Express 13, 2487 (2005).

23. M. Patterson, S. Hughes, S. Schulz, D. Beggs, T. White, L. O'Faolain, and T. Krauss, Phys. Rev. B 80, 1 (2009).

24. Q. V. Tran, S. Combrié, P. Colman, and A. De Rossi, Appl. Phys. Lett. 95, 061105 (2009).

25. P. Colman, S. Combrié, G. Lehoucq, and A. De Rossi, Opt. Express 20, 13108 (2012).

26. F. Wang, J. Jensen, and O. Sigmund, J. Opt. Soc. Am. B 28, 387 (2011).

27. E. E. Hart, A. Sóbester, K. Djidjeli, M. Molinari, K. S. Thomas, and S. J. Cox, Photon. Nanostr. Fundam. Appl. 10, 25 (2012).

28. A. Petrov, M. Krause, and M. Eich, Opt. Express 17, 8676 (2009).

29. F. Wang, J. S. Jensen, J. Mørk, and O. Sigmund, J. Opt. Soc. Am. A 29, 2657 (2012). 